\title{
Resolving Internal Structures and Composition of Biominerals: The Case of Calcitic Prisms of Mollusk Shells
}

Yannicke Dauphin ${ }^{1}$, Jean-Pierre Cuif ${ }^{1}$, Alain Brunelle ${ }^{2}$, Kadda Medjoubi ${ }^{3}$, Murielle Salomé ${ }^{4}$ and Andrea Somogyi ${ }^{3}$

${ }^{1}$ Musuem national d'Histoire naturelle, Paris, Ile-de-France, France, ${ }^{2}$ UMR LAMS, Paris, Ile-de-France, France, ${ }^{3}$ Synchrotron SOLEIL, Gif sur Yvette, Ile-de-France, France, ${ }^{4}$ ESRF, Grenoble, Rhone-Alpes, France

Biominerals are organo-mineral materials produced by living organisms. More than 50 mineral phases are identified [1]. Biominerals based on $\mathrm{CaCO}_{3}$ are found in a large number of organisms, such as mollusks, corals, echinoderms. The prismatic layers of mollusk shells are the historical references because of their large size $[2,3]$. Although they are not frequent, they are among the best-known biominerals.

Prismatic layers are, depending on the taxonomy, aragonite or calcite [4, 5]. Calcite prisms of Pteriomorpha have geometrical shape, with polygonal sections (Fig. 1A-L), but their inner structure differs. The longitudinal faces of Pinna prisms are smooth (Fig. 1C), those of Pinctada are smooth, then corrugated (Fig. 1H) [6,7], so that the growth mechanisms are not identical in these two taxa. Both species show growth layers (Fig. 1D, I) when etched using acidic or enzymatic solutions. Polarised light images show that the prisms are "monocrystalline" in Pinna, polycrystalline in Pinctada (Fig. 1E, F, J, K). Etched transverse sections clearly show the differences (Fig. 1G, 1L). Growth layers are also visible in chemical distribution maps (Fig. 1M, N), and every chemical element has its own deposition rhythm [8]. Organic envelopes surrounding the prisms of Pinna are rich in S (Fig. 1N). In Pinctada, the envelopes are poor in $\mathrm{Ca}$ associated components (Fig. 1O), rich in S (Fig. 1P), S being mainly associated with amino acids (Fig. 1Q) [9]. $\mu \mathrm{XRF}$ also shows the presence of $\mathrm{Br}$ in Pinctada (Fig. 1R), and the 3-layered structure of the envelope of Pinna (Fig. 1S). The complex structure of the interprismatic envelopes is also demonstrated using FTIR and TOF SIMS [9]. AFM images display the irregularity of the layers of the envelopes in Pinna and Pinctada (Fig. 1T, U). Although the main part of $\mathrm{CaCO}_{3}$ is crystalline (calcite), the inner structure of the prisms comprises rounded granules (Fig. 1V, W), without the angles or facets typical of non biogenic calcite. DRX and EBSD analyses have shown that the $c$ axis is parallel to the long morphological axis in Pinna, and is perpendicular in Pinctada [10]. Thus, it can be said that the morphology of biogenic calcitic prisms is determined not only by atomic structure of calcite, but largely by organic components, the presence or absence of which are drastically changing the growth mode.

Structures and compositions of calcareous biominerals differ from those of their non-biogenic counterparts. Morphological similarity of the structural units is not sufficient enough to describe a biomineral. In situ observations show structural and compositional differences, usually confirmed by analyses of the extracted organic components. The structure and composition are dependent upon the taxonomy. Prismatic layers are not the only example. The main features of the nacreous layer of Bivalvia, Gastropoda and Cephalopoda differ. Moreover, the nacreous tablets of every taxa have specific shapes, inner structure and composition. In a given layer, the shape, inner structure and composition of tablets, prisms or laths depend on the taxa, and in a given shell, they depend on the structure $[4,5,11,12]$. Only a progressive study using a large range of observations allows us to understand the organo-mineral interplay from a macro to a nanoscale. The strong control of the organisms on the shell secretion in Mollusks is not unique. Similar process exist in corals, brachiopods, etc. 

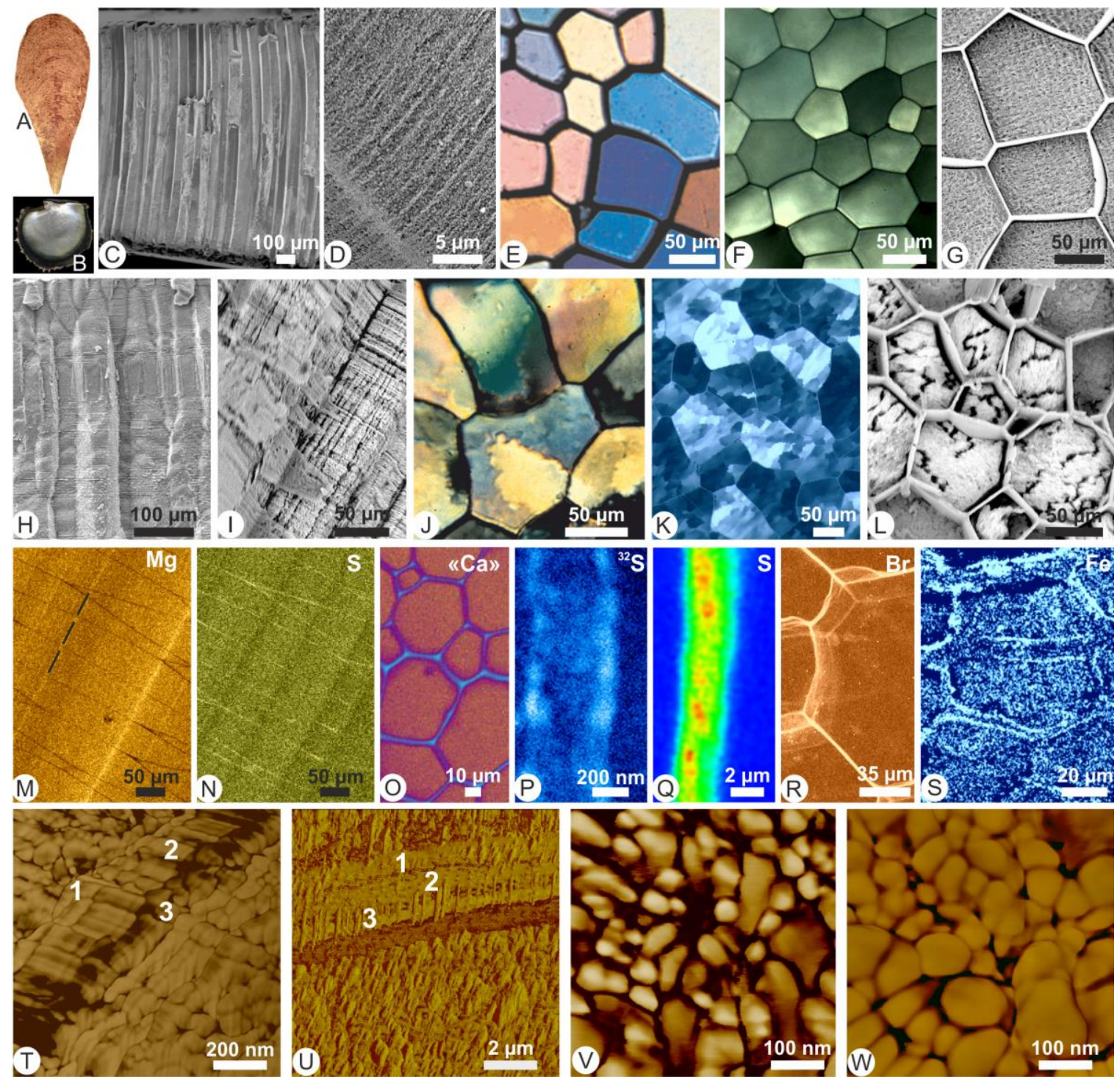

Figure 1. Figure 1. A) Outer surface of Pinna shell. B) inner surface of Pinctada. C) Fracture showing the parallel long prisms of Pinna. D) Growth layer in the prisms of Pinna. R) Thin section observed in transmitted polarized light showing the "monocrystalline" prisms of Pinna. F) Epipolarisation image of the prisms of Pinna. G) Polished and etched section showing the interprismatic organic envelopes in Pinna. H) fracture showing the smooth (top) and corrugated (bottom) surface of the prisms of Pinctada. I) Polished and etched section showing growth layers in Pinctada. J) Thin section observed in transmitted polarized light showing the polycrystalline prisms of Pinctada. K) Epipolarisation image of the prisms of Pinctada. L) Polished and etched section showing intraprismatic membranes in Pinctada. M, N) WDS distribution maps of $\mathrm{Mg}$ and $\mathrm{S}$ in Pinna. O) TOF SIMS map of Ca based ions in a transverse section of the calcitic prisms of Pinctada. P) NanoSIMS map showing 3 layers in the interprismatic envelopes of Pinctada. Q) $\mu \mathrm{XRF}$ map showing the high $\mathrm{S}$ amino acids content of the interprismatic envelopes of Pinctada. R) $\mu$ XRF bromine map of the prisms of Pinctada. S) $\mu$ XRF Fe map showing the multilayered organic envelopes of Pinna. T, U) AFM images of the interprismatic envelopes of Pinna (T) and Pinctada (V), showing 3 irregular sublayers. V, W) Rounded granules of Pinna (V) and Pinctada (W). 


\section{References}

[1] HA Lowenstam, S Weiner, On biomineralization (1989) Oxford Univ. Press, 324 p.

[2] WB Carpenter, Rep. Br. Assoc. Adv. Sci. (1844) 1-24

[3] JS Bowerbank Trans. Microsc. Soc. Lond. 1 (1844) 123-153

[4] OB Bggild, D. Kgl. Danske Vidensk. Selsk. Skr., naturvidensk. og mathem. 9 (1930), 2, 2, p. 231326

[5] JD Taylor et al., Bull. Br. Mus. nat. hist. Zool. 3 (1969), 1-125

[6] JP Cuif et al., Minerals 8 (2018), 370, doi:10.3390/min8090370

[7] JP Cuif et al., Biominerals and fossils through time (2011) Cambridge Univ. Press, 490 p.

[8] Y Dauphin et al., Marine Biology 142 (2003) 299-304

[9] Y Dauphin et al., Microsc. Microanal. 16 (2010) 91-98

[10] Y Dauphin et al., J. Struct. Biol. 205 (2019) 121-132

[11] H Mutvei, Calcif. Tiss. Res. 24 (1977), 11-18

[12] H. Nakahara, Venus 38 (1979), 3, 205-211 